\title{
Testing of a new non-equilibrium heat and moisture transfer model in porous building materials
}

\author{
Piotr Łapka ${ }^{1,{ }^{*}}$, Michal Wasik ${ }^{1}$, Eukasz Cieślikiewicz ${ }^{1}$ and Piotr Furmański ${ }^{1}$ \\ ${ }^{1}$ Institute of Heat Engineering, Faculty of Power and Aeronautical Engineering, Warsaw University of Technology, \\ 21/25 Nowowiejska St., 00-665 Warsaw, Poland
}

\begin{abstract}
In this paper the new non-equilibrium model of heat and moisture transfer in heterogenous building materials is presented and tested. The new hygro-thermal model differs from the other approaches which are based on the classical assumption of instantaneous local mechanical, thermal and hygric equilibrium between vapour and water in the pores in building materials. Instead of this assumption the model uses the finite rate of transition of moisture from the liquid to the vapour state and vice versa while still keeping mechanical and thermal equilibrium between components of the medium. The linear kinetics of this transition is applied. The assessment of the model correctness is also performed in the paper. In the first step of the testing the model predictions were successfully verified with the reference data obtained numerically. Then the model was validated using reference data obtained experimentally. Finally, the influence of volumetric mass transfer coefficient between vapour and water as well as water and vapour in pores is investigated, i.e., kinetics of the vapour-water/water-vapour transition is analysed. During the model testing traditional building material were considered (i.e., ceramic brick). However, the model may be used for investigation of hygro-thermal behaviour of bio-based materials.
\end{abstract}

\section{Introduction}

Many models for multi-dimensional analysis of heat and moisture transfer in heterogenous porous building materials have been proposed [1-9]. In such porous materials thermal phenomena significantly affect hygric processes and vice versa. Therefore, in order to correctly predict the hygro-thermal behaviour of building materials or elements of building structures (e.g., singleor multi-layered walls) the heat and mass transfer should be modelled simultaneously and in the coupled way. The most advanced models applied for prediction of heat and moisture transfer in porous media are called Heat, Air and Moisture (HAM) models. Sometimes HAM models are combined with either the Building Energy Simulation (BES) or Computational Fluid Dynamics (CFD) simulation tools [10]. These complex models account not only for heat and moisture transfer in building materials or building structure elements (e.g., walls) but also for effect of moisture presence on the energy consumption in buildings or heat and moisture transfer in flowing air which is in the contact with the considered sample of material. Usually, the HAM-CFD models were implemented applying the commercial CFD software like ANSYS Fluent or COMSOL Multiphysics $[3,4,6]$.

The mentioned models are based on the classical hygro-thermal transfer model which assumes mechanical and thermal equilibrium in the porous medium. What is more important, its also assumes that vapour and water which fill the pores are in the equilibrium, i.e., for a given local thermal state the saturation conditions for the vapour are achieved instantaneously (i.e., hygric equilibrium is attained). However, in some materials, i.e., bio-based materials like lime-based hempcretes, cements compounds and concreates, the assumption about hygric equilibrium between vapour and water was shown not valid $[11,12]$.

Recently new models which deal with nonequilibrium moisture transfer in the heterogenous porous building materials have been proposed [11-16]. These models assumed finite rate of mass transfer between vapour and water and vice versa. It was shown that if the proper kinetics for describing the transformation of moisture from gaseous to liquid state and vice versa is chosen these models may be applied to account for combined heat and moisture transfer in bio-based building materials in which classical assumption about hygric equilibrium between gaseous and liquid moisture is not valid $[11,12]$.

In the paper the model proposed in [13-16] is tested. This model assumes the linear kinetics of transition of moisture from gaseous to liquid state and vice versa. Its verification and validation for the classical building material (i.e., ceramic brick) is presented. Then influence of volumetric mass transfer coefficient which describes the dynamics of transition between gaseous and liquid moisture and vice versa in pores of building material investigated.

\footnotetext{
$\overline{\text { * Corresponding author: piotr.lapka@pw.edu.pl }}$
} 


\section{New non-equilibrium heat and moisture transfer model}

\subsection{Mathematical formulation}

The new non-equilibrium model of hygro-thermal behaviour of porous building materials consists of three coupled equations which account for vapour, water and energy balance, respectively. These equations are the following:

$$
\begin{gathered}
\frac{\partial}{\partial t}\left(\varepsilon_{g} \rho_{v}\right)-\nabla \cdot\left(D_{v, e f} \nabla \rho_{v}\right)=\dot{m}_{l v} \\
\frac{\partial}{\partial t}\left(\varepsilon_{l} \rho_{l}\right)+\nabla \cdot\left(K_{l} \nabla p_{c}\right)=-\dot{m}_{l v} \\
\frac{\partial}{\partial t}\left[(\rho c)_{e f} T\right]+\nabla \cdot\left(h_{l} K_{l} \nabla p_{c}\right)+ \\
-\nabla \cdot\left(h_{v} D_{v, e f} \nabla \rho_{v}\right)=\nabla \cdot\left(k_{e f} \nabla T\right)-\dot{m}_{l v} \Delta h_{l v}
\end{gathered}
$$

where: subscripts $g, l$ and $v$ denote moist air (the mixture of dry air and vapour), water and vapour, respectively and the symbol $(\rho c)_{e f}$ represents the effective heat capacity of the porous media, $D_{v-a, e f}-$ effective water vapour diffusivity in the porous media, $h$ - enthalpy, $k_{e f}$ - effective thermal conductivity of the porous media, $K_{l}$ - permeability of the porous medium for liquid water, when the water vapour is present in pores, $l v-$ volumetric intensity of liquid evaporation/ vapour condensation, $p_{c}$ - capillary pressure, $t-$ time, $T$ - temperature, $\Delta h_{l v}$ - latent heat of evaporation, $\varepsilon-$ volume fraction and $\rho-$ density. Closing relationships and formulae for effective properties of the porous media which are required in eq. (1)-(3) are presented later (see section 2.2).

In this paper the linear kinetics of evaporation/condensation in the pores of building material is assumed and is given by the following relationship:

$$
\dot{m}_{l v}=\left\{\begin{array}{l}
h_{v l} a_{s} \frac{\varepsilon_{l}}{\varepsilon_{p}}\left(\rho_{v, s a t}-\rho_{v}\right)-\text { for evaporation } \\
h_{v l} a_{s}\left(\rho_{v, s a t}-\rho_{v}\right)-\text { for condensation }
\end{array}\right.
$$

where: subscripts $p$ and sat denote pores and saturation conditions, the symbol $a_{s}$ is the pores area pre unit volume of the medium, $h_{v l}$ - volumetric mass transfer coefficient which describes dynamics of the transition from vapour to water and vice versa in the pores. The eq. (4) contains the water to pore volume fraction ratio $(l / p)$ which models the part of the pores volume occupied by water.

The boundary conditions for eq. (1) and (2) at the walls which are contacting with flowing air are dependent on the state of moisture at the boundary. If moisture in the pendular state (continuous) is present at the wall, the evaporation from the surface takes place. For this case the water saturation is assumed to be higher than the minimal saturation for water in the pendular form, i.e., $s>s_{\min }=0.25$, and the vapour and water fluxes at the wall are the following:

$$
\begin{gathered}
\left(-D_{v, e f} \nabla \rho_{v}\right)_{w}=0 \\
\left(K_{l} \nabla p_{c}\right)_{w}=h_{m}\left(\frac{\rho_{v, w}}{\rho_{g, w}}-Y_{a m b}\right)
\end{gathered}
$$

where: subscripts $a m b$ and $w$ denote the ambient and wall, the symbol $h_{m}$ is the convective mass transfer coefficient at the wall and $\mathrm{Y}$ - mass fraction of vapour.

For the second case, when water saturation is below minimal saturation for water in the pendular form, i.e., $s<s_{\min }=0.25$ (water is in the funicular form) the vapour and water fluxes are the following:

$$
\begin{gathered}
\left(-D_{v, e f} \nabla \rho_{v}\right)_{w}=h_{m}\left(\frac{\rho_{v, w}}{\rho_{g, w}}-Y_{a m b}\right) \\
\left(K_{l} \nabla p_{c}\right)_{w}=0
\end{gathered}
$$

The boundary conditions for energy equation, eq. (3), assumes convective and thermal radiative heat transfer from the external wall to the surroundings and is in the following form:

$$
\begin{aligned}
& \left(-k_{e f} \nabla T\right)_{w}=h_{t}\left(T_{w}-T_{a m b}\right)+ \\
& \frac{\sigma\left(T_{w}^{4}-T_{a m b, r a d}^{4}\right)}{\frac{1}{e_{w}}+\frac{1}{e_{a m b}}-1}+\Delta h_{l v} K_{l} \nabla p_{c}
\end{aligned}
$$

where: the subscript rad refers to thermal radiation, the symbol $e$ is the emissivity, $h_{t}$ - convective heat transfer coefficient at the wall and $\sigma$ - Stefan-Boltzmann constant.

\subsection{Additional relationship required for closing the system of equations}

The new non-equilibrium model defined by eq. (1)(3) requires closing relations and formulae for effective hygro-thermal properties of considered porous material. Some of these properties depends on the material type and its morphology (microstructure) and are measured. Therefore, in this paper they were assumed for ceramic brick after Steeman et al. [17]. To solve system of governing equations, eq. (1)-(3), the following relations are used:

Pores area per unit volume:

$$
a_{s}=\frac{6}{d_{a v}\left(1-\varepsilon_{s}\right)}
$$

Effective heat capacity:

$$
(\rho c)_{e f}=\varepsilon_{s} \rho_{s} c_{s}+\varepsilon_{l} \rho_{l} c_{l}+\varepsilon_{g} \rho_{a} c_{p, a}+\varepsilon_{g} \rho_{v} c_{p, v}
$$


Vapour diffusivity in pores:

$$
D_{v, e f}=\frac{2.61 \cdot 10^{-5} M_{v}\left(1-\frac{W}{W_{c a p}}\right)}{C_{d r y} B T\left[0.503\left(1-\frac{W}{W_{c a p}}\right)^{2}+0.497\right]}
$$

Specific enthalpies:

$$
\begin{aligned}
& h_{l}=c_{l}\left(T-T_{r e f}\right) \\
& h_{s}=c_{s}\left(T-T_{r e f}\right) \\
& h_{v}=c_{p, v}\left(T-T_{r e f}\right)+\Delta h_{v l}
\end{aligned}
$$

Effective thermal conductivity of the moist brick:

$$
k_{e f}=k_{s}+0.0047 \mathrm{~W}
$$

Water permeability in the brick:

$$
K_{l}=\frac{1.1437 \cdot 10^{-9}}{\left[1+\left(1.76 \cdot 10^{-5} p_{c}\right)^{4.3}\right]^{1.6}}
$$

Vapour saturation pressure:

$$
p_{v, s a t}=614.3 \exp \left(17.06 \frac{T-273.15}{T-40.25}\right)
$$

Modified saturation pressure:

$$
p_{v, s a t}^{*}=p_{v, s a t} \exp \left(-\frac{p_{c} M_{v}}{\rho_{l} B T}\right)
$$

Liquid saturation:

$$
s=\frac{\varepsilon_{l}}{1-\varepsilon_{s}}
$$

Retention curve which was applied to find the capillary pressure:

$$
\begin{aligned}
& W\left(p_{c}\right)= \\
& =W_{\text {cap }}\left\{\begin{array}{l}
0.846\left[1+\left(1.394 \cdot 10^{-5} p_{c}\right)^{4}\right]^{-0.75}+ \\
0.154\left[1+\left(0.9011 \cdot 10^{-5} p_{c}\right)^{1.69}\right]^{-0.408}
\end{array}\right\}
\end{aligned}
$$

Volumetric moisture content:

$$
W=\rho_{l} \varepsilon_{l}+\rho_{v} \varepsilon_{g}
$$

Water vapour density for saturation conditions:

$$
\rho_{v, s a t}=\frac{p_{v, s a t}^{*} M_{v}}{B T}
$$

where: subscripts $a$ and $s$ denote dry air and solid component (dry brick), respectively, the symbol $B$ is the universal gas constant, $c-$ specific heat, $c_{p}-$ specific heat at constant pressure, $C_{d r y}$ - water vapour resistance diffusion factor, $d_{a v}$ - average pore diameter, $M_{v}-$ molecular mass of vapour, $T_{r e f}$ - reference temperature for the enthalpy origin and $W_{c a p}$ - capillary moisture content.

If required, densities and pressures of dry and moist air as well as vapour were calculated applying the ideal gas relationship.

\subsection{Numerical implementation}

The new non-equilibrium model is implemented in the framework of the commercial software ANSYS Fluent applying its advance customisation functions. Governing equations, eq. (1)-(3), with boundary conditions and all closing relationships are implemented in the ANSYS Fluent using User Define Function (UDF), User Define Scalar (UDS) and User Define Memory (UDM). For simulations presented in this paper the $2 \mathrm{D}$ version of the model is utilised but it is currently also implemented in the 3D space. The model implementation using commercial software allows for fast and easy modifications of the computational geometry and meshes, e.g., even very complex geometries may be easily generated and applied in simulations. Moreover, using commercial software will enable planned future coupling of the model with CFD simulation of the flowing ambient air.

\section{Testing of the model}

For the testing the presented model is configured in the following way. The rectangular $2 \mathrm{D}$ sample which represents the ceramic brick is considered. The sample is in the contact with drying air at the top boundary so at this wall the boundary conditions given by eq. (5)-(9) are imposed. The side and bottom walls are assumed adiabatic and impermeable. For these boundary conditions configuration, the $1 \mathrm{D}$ heat and moisture transfer occurs in the considered sample. Therefore, its width may be assumed arbitrary (i.e., $1 \mathrm{~cm}$ was selected for the testing), while its thickness was $3 \mathrm{~cm}$. This also affects the way the computational mesh was created, i.e., 60 elements along the brick thickness were generated, while only 1 element was used along the brick width. What is more, during simulations the time step size was equal to $\Delta t=5 \mathrm{~s}$, which allowed for carrying out stable calculations. Moreover, the sensitivity of the obtained results to grid and time step size were performed. The analysis proved that for number of elements and time step size within the range from 30 to 120 and from $1 \mathrm{~s}$ to $10 \mathrm{~s}$, respectively, the obtained results were independent on these parameters.

The brick parameters assumed in simulations are presented in Table 1 and are the same as in [18]. 
Table 1. Hygro-thermal properties assumed during simulations [13].

\begin{tabular}{|c|c|c|}
\hline Property & $\begin{array}{c}\text { Symbol and } \\
\text { unit }\end{array}$ & Value \\
\hline Universal gas constant & $B(\mathrm{~J} / \mathrm{kmol} / \mathrm{K})$ & 8314.0 \\
\hline $\begin{array}{l}\text { Dry air specific heat at } \\
\text { constant pressure }\end{array}$ & $c_{p, a}(\mathrm{~J} / \mathrm{kg} / \mathrm{K})$ & 1005.0 \\
\hline Water specific heat & $c_{l}(\mathrm{~J} / \mathrm{kg} / \mathrm{K})$ & 4192.1 \\
\hline Brick specific heat & $c_{s}(\mathrm{~J} / \mathrm{kg} / \mathrm{K})$ & 840.0 \\
\hline $\begin{array}{l}\text { Vapour specific heat at } \\
\text { constant pressure }\end{array}$ & $c_{p, v}(\mathrm{~J} / \mathrm{kg} / \mathrm{K})$ & 1875.2 \\
\hline $\begin{array}{l}\text { Water vapour resistance } \\
\text { diffusion factor }\end{array}$ & $C_{d r y}$ & 24.79 \\
\hline Average pore diameter & $d_{a v}(\mathrm{~m})$ & $1.6 \cdot 10^{-5}$ \\
\hline $\begin{array}{l}\text { Volumetric mass transfer } \\
\text { coefficient between vapour } \\
\text { and water in porous } \\
\text { medium }\end{array}$ & $h_{v l}(\mathrm{~m} / \mathrm{s})$ & $\begin{array}{c}2.0 \cdot 10^{-5} \\
\text { (for } \\
\text { verification) } \\
2.5 \cdot 10^{-5} \\
\quad \text { (for } \\
\text { validation) }\end{array}$ \\
\hline Brick thermal conductivity & $k_{s}(\mathrm{~W} / \mathrm{m} / \mathrm{K})$ & 1.0 \\
\hline Dry air molecular mass & $M_{a}(\mathrm{~kg} / \mathrm{kmol})$ & 28.86 \\
\hline Vapour molecular mass & $M_{v}(\mathrm{~kg} / \mathrm{kmol})$ & 18.0 \\
\hline Capillary moisture content & $W_{\text {cap }}\left(\mathrm{kg} / \mathrm{m}^{3}\right)$ & 130.0 \\
\hline $\begin{array}{l}\text { Volume fraction of pores } \\
\text { (brick porosity) }\end{array}$ & $p$ & 0.13 \\
\hline Latent heat of evaporation & $h_{l v}(\mathrm{~J} / \mathrm{kg})$ & $2.5 \cdot 10^{6}$ \\
\hline Water density & $l\left(\mathrm{~kg} / \mathrm{m}^{3}\right)$ & 1000.0 \\
\hline Brick density & $s\left(\mathrm{~kg} / \mathrm{m}^{3}\right)$ & 2087.0 \\
\hline
\end{tabular}

Parameters of boundary conditions at the top wall were assumed after Belleghem [18] and were the following: the temperature and relative humidity of drying air of $T_{a m b}=23.8^{\circ} \mathrm{C}$ and $\varphi_{a m b}=44 \%$, respectively, the heat and mass transfer coefficient equal to $h_{t}=22.5 \mathrm{~W} / \mathrm{m}^{2} / \mathrm{K}$ and $h_{m}=0.0258 \mathrm{~kg} / \mathrm{m}^{2} / \mathrm{s}$, respectively, the top wall and surrounding surfaces emissivities of $e_{w}=0.93$ and $e_{a m b}=0.97$, respectively and the surroundings radiation temperature of $T_{a m b, r a d}=23.3^{\circ} \mathrm{C}$. The initial conditions were the following: the uniform temperature and volumetric moisture content in the sample equal to $T_{0}=23.8^{\circ} \mathrm{C}$ and $W_{0}=126.1 \mathrm{~kg} / \mathrm{m}^{3}$, respectively. This initial amount of moisture corresponded to water saturation equal to $s_{0}=97 \%$.

The volumetric mass transfer coefficient in porous medium between vapour and water and vice versa (i.e., the vapour-water/water-vapour transition kinetics constant) varied from case to case. Its value was adjusted to obtain better matching of the obtained results with the reference data. So, the $h_{l v}$ is here used as the model tuning parameter. During verification its value was assumed equal to $h_{l v}=2.0 \cdot 10^{-5} \mathrm{~m} / \mathrm{s}$, while during validation it was increased to $h_{l v}=2.5 \cdot 10^{-5} \mathrm{~m} / \mathrm{s}$.

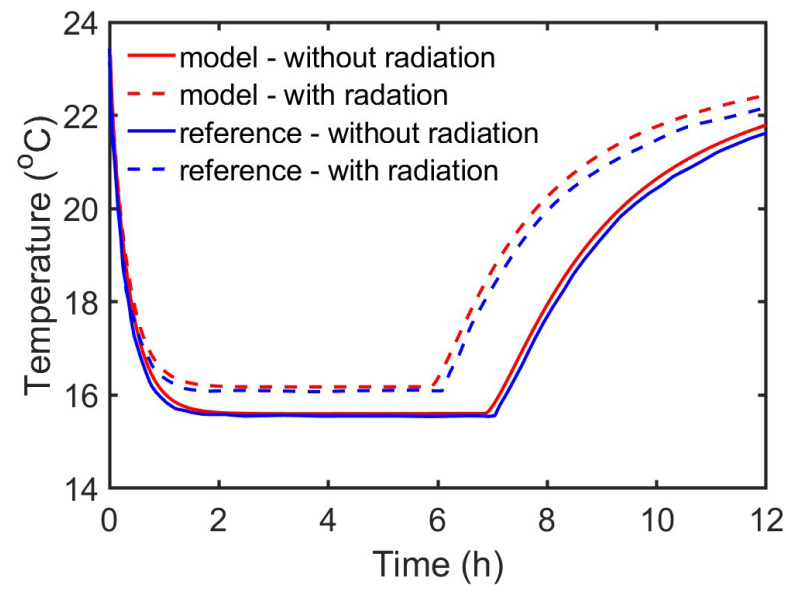

Fig. 1. Temporal variations of temperature in the brick at $1 \mathrm{~cm}$ depth from the top surface (red line - new non-equilibrium model and blue line - reference numerical data from [19]).

\subsection{Verification}

In the first step of the testing of the new approach the verification of the model predictions with numerical results presented in [19] has been carried out. The boundary conditions and material properties were as described above. Two scenarios were considered. In the first one the brick was in the convective and radiative contact with the surroundings, while in the second one the radiative heat transfer was neglected and the brick was only in the convective contact with the surroundings (i.e., the second term on the right hand side of eq. (9) was omitted). The results of the comparison are shown in Fig. 1. One can see that temporal variations of the temperature in a brick in a point located $1 \mathrm{~cm}$ below the top surface predicted with present model and obtained by the model of Belleghem [19] match each other very well for both scenarios. Similar matching was obtained for the point on $2 \mathrm{~cm}$ depth.

\subsection{Validation}

In the second step of the testing of the new nonequilibrium model the validation of the model predictions with experimental measurements from [19] has been carried out. The ceramic brick hygro-thermal parameters as well as initial condition for the validation were the same as during verification case. The geometry, mesh and time step also remained unchanged. The boundary conditions at the top wall has been reformulated, i.e., the smoothing function was applied to determined water and vapour transfer from brick surface to the surroundings. After reformulation these boundary conditions were the following:

$$
\left(-D_{v, e f} \nabla \rho_{v}\right)_{w}=[1-f(s)] h_{m}\left(\frac{\rho_{v, w}}{\rho_{g, w}}-Y_{a m b}\right)
$$




$$
\left(K_{l} \nabla p_{c}\right)_{w}=f(s) h_{m}\left(\frac{\rho_{v, w}}{\rho_{g, w}}-Y_{a m b}\right)
$$

and the smoothing function was the following:

$$
f(s)=\sin ^{2}\left(\frac{\pi s}{2}\right)
$$

The new formulation of the boundary conditions allowed to avoid the step change of the amount of liquid evaporating from the top surface with the change of the water saturation.

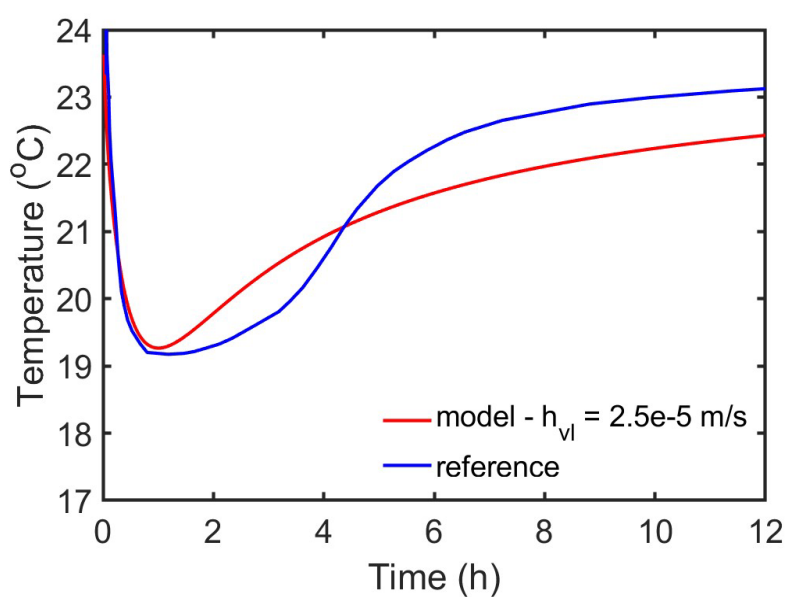

Fig. 2. Temporal variations of temperature in the brick at $1 \mathrm{~cm}$ depth from the top surface (red line - new non-equilibrium model and blue line - reference experimental data from [19]).

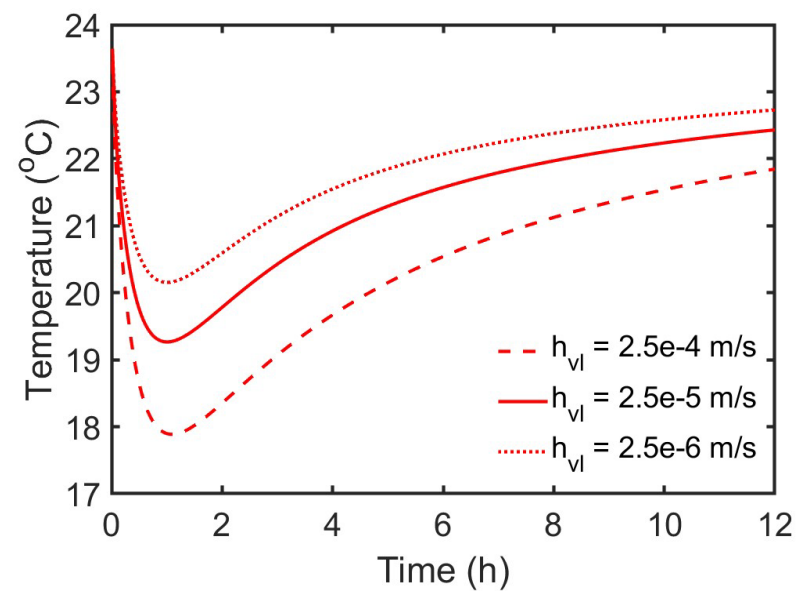

Fig. 3. Temporal variations of temperature in the brick at $1 \mathrm{~cm}$ depth from the top surface for various values of the volumetric mass transfer coefficient in porous medium between vapour and water and vice versa.

The comparison of the obtained results (i.e., temperature distributions at $1 \mathrm{~cm}$ depth from the top surface) with the experimental data from [19] is presented in Fig. 2. The matching of the results in not perfect but more or less similar temperature distributions were obtained. Similar distributions and matching were obtained for the point located deeper, i.e., $2 \mathrm{~cm}$ bellow the top surface. The shorter temperature plateau and slower rise of calculated temperature than measured experimentally is the effect of the applied switching function. In this paper different smoothing function have been tested, e.g., linear or exponential. But for the one given by eq. (24) the best matching of the measured and predicted results have been obtained. Moreover, it seems that the smoothing function, for which better agreement of the results will be obtained, may be found. Such search is undergoing.

\subsection{Investigation of influence of volumetric mass transfer coefficient between vapour and water in porous medium}

In the last step of the testing of the new non-equilibrium model the investigation of the influence of variation of the volumetric mass transfer coefficient in porous media between vapour and water and vice versa was carried out. In these simulations the model setup was the same as for validation, expect for the value of $h_{l v}$. In this section, three values of the volumetric mass transfer coefficient were assumed, i.e., $h_{l v}=2.5 \cdot 10^{-6} \mathrm{~m} / \mathrm{s}$, $h_{l v}=2.5 \cdot 10^{-5} \mathrm{~m} / \mathrm{s}$ and $h_{l v}=2.5 \cdot 10^{-4} \mathrm{~m} / \mathrm{s}$. The temperature distributions at depth of $1 \mathrm{~cm}$ measured for the top wall are shown in Fig. 3. Similar distributions were obtained for points located deeper in the brick. Significant influence of $h_{l v}$ on the obtained temperature distributions is observed. If value of $h_{l v}$ increases the faster evaporation of the liquid moisture is observed and therefore more heat is consumed for this process - see dashed line in Fig. 3. This leads to the higher drop of temperature of building material. For lower values of $h_{l v}$ the less vapour is generated which refers to the less heat absorbed in the phase change process and lower temperature drop - see dotted line in Fig. 3.

\section{Conclusion}

In this paper the new non-equilibrium model of heat and moisture transfer in porous building materials has been presented and tested. The model assumed finite rate of translation of moisture from gaseous to liquid state and vice versa. In this way the assumption related to the hygric equilibrium in the wet porous medium which is commonly found in other models presented in the literature was released. However, the mechanical and thermal equilibrium have still been kept.

The presented model was thoroughly tested. The model predictions were compered with the numerically obtained reference results and with the experimental measurements. The good agreement of the obtained results and the reference data has been obtained. Moreover, analysis of influence of variation of the volumetric mass transfer coefficient in porous media between vapour and water and vice versa was performed. The $h_{l v}$ was found to have significant effect on the temperature distribution in the sample of building material.

Although the model testing was performed for the classical building material (i.e., ceramic brick), it was shown that the model has potential to be applied for analysis of the bio-based building materials, i.e., different kinetics of the vapour-water/water-vapour 
transition may be applied in the model and these kinetics significantly affects the obtained temperature distributions. In these materials the hygric equilibrium assumption is not valid. But before the application of the model for analysis of hygro-thermal behaviour of such materials its verification and validation using numerical and experimental data obtained for the bio-based materials is required. Such studies are planned.

This work was supported from European Union within the European Regional Development Fund under project no. POIR.04.01.02-00-0099/16 "Development of innovative technology of drying and moisture sealing of masonry walls, DryWall" granted by the National Centre for Research and Development (Poland).

\section{References}

1. S. Whitaker, Adv. Heat Transfer 13, 119, (1977)

2. P. Salagnac, P. Glouannec, D. Lecharpentier, Int. J. Heat Mass Transf. 47, 4479 (2004)

3. M. Van Belleghem, H.-J. Steeman, M. Steeman, A. Janssens, M. De Paepe, Build. Environ. 45, 2485 (2010)

4. M. Van Belleghem, M. Steeman, H. Janssen, A. Janssens, M. De Paepe, Build. Environ. 81, 340 (2014)

5. C. Belleudy, M. Woloszyn, M. Chhay, M. Cosnier, Int. J. Heat Mass Transf. 95, 453 (2016)

6. B.M. Janetti, L.P.M. Colombo, F. Ochs, W. Feist, Energy Build. 166, 550 (2018)

7. R. Allam, N. Issaadi, R. Belarbi, M. El-Meligy, A. Altahrany, Heat Mass Transfer 54, 1579 (2018)

8. T. Busser, J. Berger, A. Piot, M. Pailha, M. Woloszyn, Dry. Technol. 37, 1363 (2019)

9. M. Seredyński, P. Furmański, P. Łapka, M. Wasik, Ł. Cieślikiewicz, K. Pietrak M. Kubiś, T. Wiśniewski, M. Jaworski, IOP Conf. Ser. Mater. Sci. Eng. 660, 012023 (2019)

10. H.J. Steeman, A. Janssens, J. Carmeliet, M. De Paepe, Build. Environ. 44, 572 (2009)

11. N. Reuge, S. Moissette, M. Bart, F. Collet, C. Lanos, Transp. Porous Media 128, 821 (2019)

12. N. Reuge, F. Collet, S. Pretot, S. Moisette, M. Bart, O. Style, A. Shea, C. Lanos, Constr. Build. Mater. 240, 117928 (2020)

13. P. Łapka, M. Wasik, P. Furmański, M. Seredyński, Ł. Cieślikiewicz, K. Pietrak, M. Kubiś, T. Wiśniewski, M. Jaworski, MATEC Web Conf. 240, 01022 (2018)

14. M. Wasik, Ł. Cieślikiewicz, P. Łapka, P. Furmański, M. Kubiś, M. Seredyński, K. Pietrak, T. Wiśniewski, M. Jaworski, AIP Conf. Proc. 2078, 020106 (2019)

15. M. Seredyński, M. Wasik, P. Łapka, P. Furmański, Ł. Cieślikiewicz, K. Pietrak, M. Kubiś, T. Wiśniewski, M. Jaworski, E3S Web Conf. 128, 06008 (2019)
16. M. Seredyński, M. Wasik, P. Łapka, P. Furmański, Ł. Cieślikiewicz, K. Pietrak, M. Kubiś, T. Wiśniewski, M. Jaworski, Energies 13, 214 (2020)

17. H.-J. Steeman, M. Van Belleghem, A. Janssens, De M. Paepe, Build. Environ. 44, 2176-2184 (2009)

18. M. Van Belleghem, B. Ameel, A. Janssens, M. De Paepe, Proc. 8th International Conference on Heat Transfer, Fluid Mechanics and Thermodynamics, HEFAT2011, 455-463 (2011)

19. M. Van Belleghem, Modelling coupled heat and moisture transfer between air and porous materials for building applications, $\mathrm{PhD}$ thesis, Ghent University, Belgium (2013) 